\title{
Aphasia and a Dual-Stream Language Model in a 4-Year-Old Female with Landau-Kleffner Syndrome
}

\author{
${ }^{1}$ Division of Neurology, National Center for Child Health and \\ Development, Tokyo, Japan \\ 2 Department of Laboratory Medicine, University of Tokyo, Tokyo, \\ Japan \\ 3 Department of Pediatrics, Shimada Ryoiku Medical Center for \\ Challenged Children, Tokyo, Japan
}

Michiko Kawai ${ }^{11}$ Yuichi Abe ${ }^{1}$ Masato Yumoto ${ }^{2}$ Masaya Kubota ${ }^{1,3(1)}$

\author{
Address for correspondence Michiko Kawai, MD, Division of \\ Neurology, National Center for Child Health and Development, Okura \\ 2-10-1 Setagaya, 157-0074, Tokyo, Japan \\ (e-mail: kawai-mc@ncchd.go.jp).
}

Neuropediatrics 2022;53:295-298.

\begin{abstract}
Keywords

- Landau-Kleffner syndrome

- aphasia

- dual-stream model

- epileptogenic zone

Landau-Kleffner syndrome (LKS) is a rare neurological disorder characterized by acquired aphasia. LKS presents with distinctive electroencephalography (EEG) findings, including diffuse continuous spike and wave complexes (CSW), particularly during sleep. There has been little research on the mechanisms of aphasia and its origin within the brain and how it recovers. We diagnosed LKS in a 4-year-old female with an epileptogenic zone located primarily in the right superior temporal gyrus or STG (nondominant side). In the course of her illness, she had early signs of motor aphasia recovery but was slow to regain language comprehension and recover from hearing loss. We suggest that the findings from our patient's brain imaging and the disparity between her recovery from expressive and receptive aphasias are consistent with the dual-stream model of speech processing in which the nondominant hemisphere also plays a significant role in language comprehension. Unlike aphasia in adults, the righthemisphere disorder has been reported to cause delays in language comprehension and gestures in early childhood. In the period of language acquisition, it requires a process of understanding what the words mean by integrating and understanding the visual, auditory, and contextual information. It is thought that the right hemisphere works predominantly with respect to its integrating role.
\end{abstract}

\section{Introduction}

Landau-Kleffner syndrome (LKS) is a rare neurological disorder characterized by acquired aphasia. LKS presents with distinctive electroencephalography (EEG) findings, including diffuse continuous spike and wave complexes (CSW), particularly during sleep. There has been little research on the mechanisms of the aphasia and its origin within the brain. CSW occurs bilaterally in $80 \%$ of LKS patients and unilaterally in only 20\%; however, even unilateral cases exhibit spikes of LKS in both hemispheres synchronously. ${ }^{1}$

received

April 14, 2021

accepted after revision

July 8,2021

published online

December 1, 2021
We diagnosed LKS in a 4-year-old female with an epileptogenic zone located primarily in the right STG (nondominant side). In the course of her illness, she had early signs of motor aphasia recovery but was slow to regain language comprehension and recover from hearing loss. We suggest that the findings from our patient's brain imaging and the disparity between her recovery from expressive and receptive aphasias are consistent with the dual-stream model of speech processing in which the nondominant hemisphere also plays a significant role in language comprehension.

(c) 2021. Thieme. All rights reserved. Georg Thieme Verlag KG, Rüdigerstraße 14, 70469 Stuttgart, Germany
DOI https://doi.org/ 10.1055/s-0041-1733983. ISSN 0174-304X. 

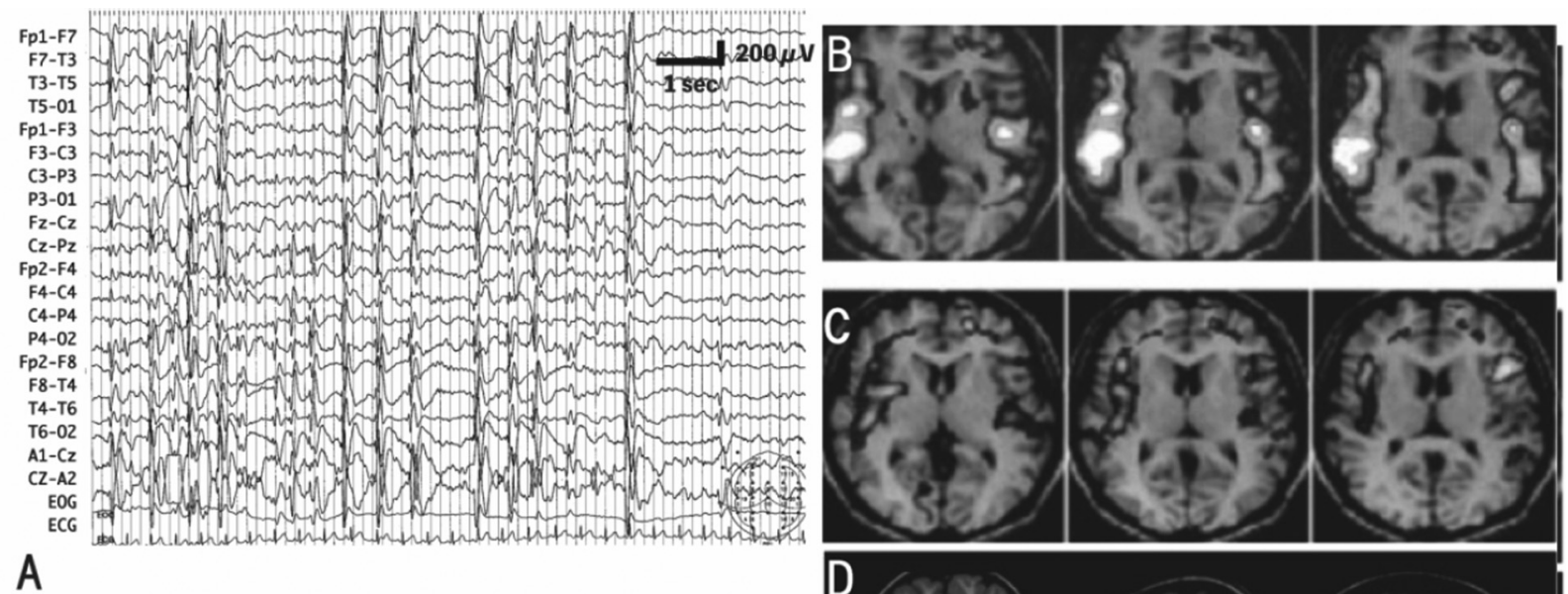

A

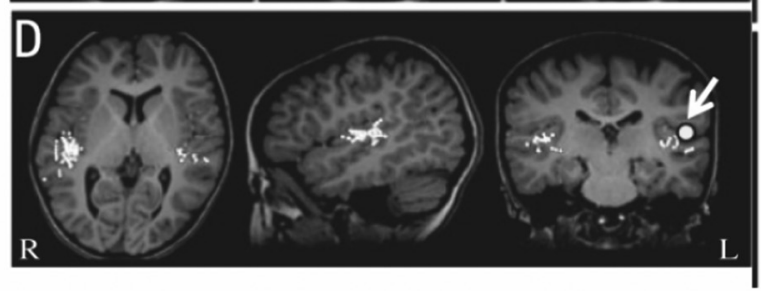

Fig. 1 (A) The scalp EEG during an acute phase revealed diffuse Rolandic CSW at central and parietal bilaterally synchronized or right-hemisphere independently. (B,C) ECD-SPECT at relapse phase (B) showed hyperperfusion in the bilateral anterior temporoparietal area with right-hemisphere dominance. This hyperperfusion disappeared in the recovery phase (C). (D) The equivalent current dipoles for MEG spikes (white dots) clustered mainly in the bilateral superior temporal gyri with right-hemisphere dominance (AEF) to 1-kHz tone burst (N100m) was absent at the right auditory cortex, whereas it remained normal at the left (large white circle, arrow).

\section{Case Presentation}

A 4-year-old, right-handed female presented with frequent (one to five times an hour) paroxysmal blinking and impaired consciousness. Her health and psychomotor development history had been normal until age three; however, her verbal articulation had begun to slowly regress 6 months before the onset of blinking episodes. An EEG revealed diffuse frequent Rolandic spike and wave synchronously in both hemispheres or independently in the right hemisphere mainly in the central and parietal region (-Fig. 1A). The seizures with rapid eye blinking and impaired consciousness during awake were soon disappeared by administration of levetiracetam ( $25 \mathrm{mg} / \mathrm{kg} / \mathrm{d}$ ); however, the EEG remained unchanged. The patient's speech ability gradually worsened, and she developed aphasia within 3 months.

A long-term EEG revealed diffuse CSW during both waking and sleeping states. CSW index was $86.1 \%$. No abnormalities were seen during brain magnetic resonance imaging, and auditory brainstem responses were normal. Consequently, we diagnosed her with LKS. The exome sequencing test was negative including GRIN2A. Based on the results of the neuroimagings, we expect that the etiology of the syndrome is not structural, but functional, with genetic factors yet to be proven.

The Wechsler Preschool and Primary Scale of IntelligenceIII (WIPPSI-III) test at age 4 years and 3 months was 80 for Full scale intelligence quotient (IQ), 55 for verbal IQ, 116 for performance IQ, and 80 for processing speed IQ.
Immediately after the diagnosis of LKS, we treated her with two courses of steroid pulse therapy (intravenous methylprednisolone [IVMP]) and clonazepam $(0.04 \mathrm{mg} / \mathrm{kg} /$ d). Fortunately, the CSW was effectively suppressed by these treatments.

For the evaluation of language function over time, we used the Sign-Significate method (S-S method) of the National Rehabilitation Center for Persons with Disabilities, which is commonly used in Japan. Before starting treatment, most of her vocalizations were nonsensical and it was difficult to understand only auditory instructions, however, within a month of the CSW being suppressed, it became possible to call some of the items depicted on the picture cards. (One out of 16 tasks was answered correctly in terms of accurate object names, and five tasks were comprehensible to the examiner using infant words such as "meow" for cat and "vroom-vroom" for car). On the contrary, there was no noticeable improvement in auditory instruction comprehension, and she did not respond to listening task at all.

Three months later, small spikes again appeared in the right hemisphere, and the EEG examination showed a recurrence of CSW. Her expressive speech disorder was less severe at relapse compared with the first episode.

Examination by ${ }^{99} \mathrm{~m}$ Tc-ethylcysteine dimer single-photon emission computed tomography (ECD-SPECT) at relapse showed hyperperfusion in the bilateral anterior temporoparietal area with right-hemisphere dominance (-Fig. 1B).

The patient's ability to respond to sound was tested with the use of an MEG at relapse, which showed equivalent current 


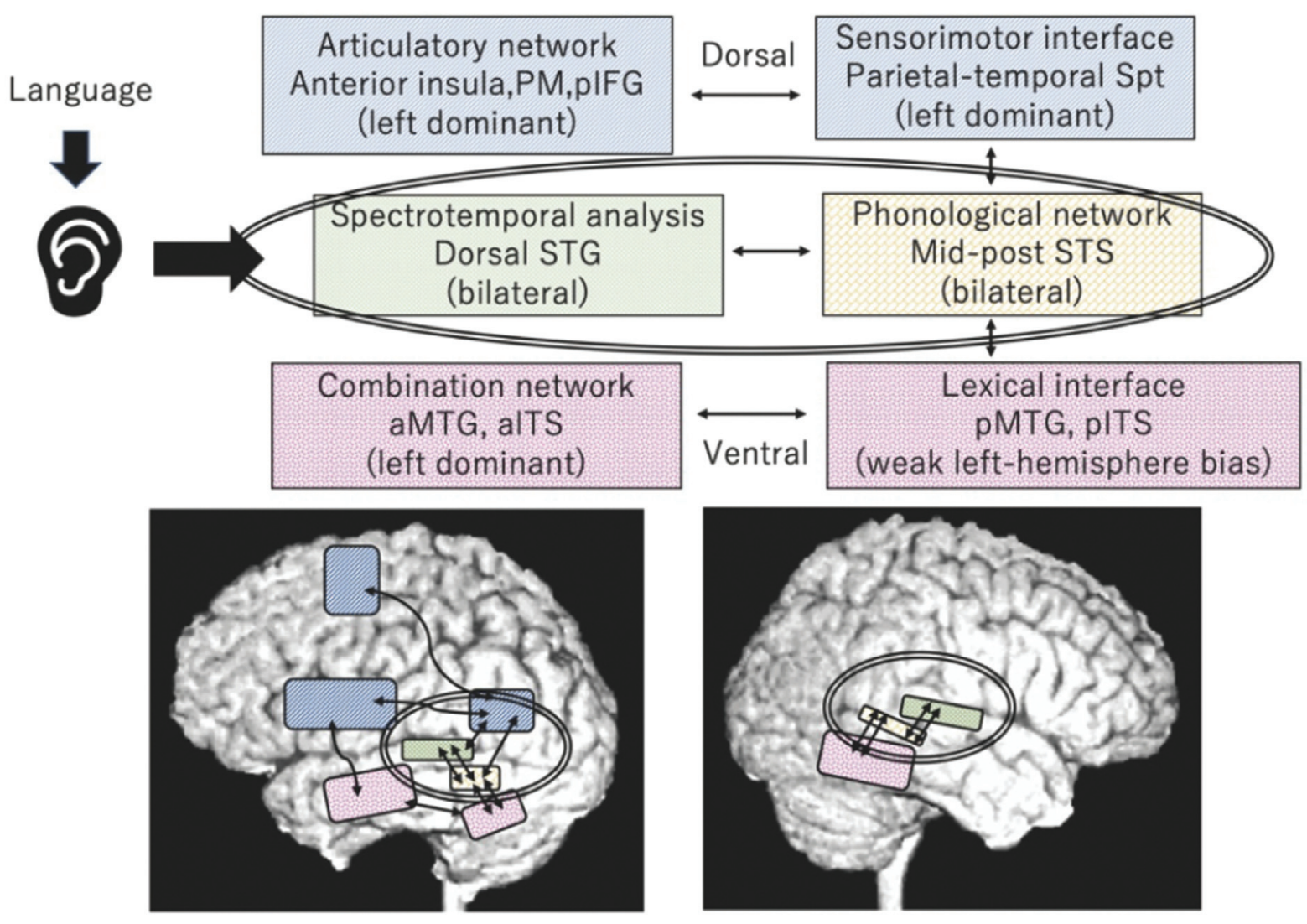

Fig. 2 The dual-stream model of speech processing, with some simplification, based on the study by Hickok and Poeppel.2 The early stage of speech processing occurs bilaterally in the auditory cortex on the dorsal STG and STS (surrounded by an ellipse), then divides into ventral and dorsal streams. IFG, inferior frontal gyrus; ITS, inferior temporal sulcus; MTG, middle temporal gyrus; PM, premotor; SPT, Sylvian parietaltemporal; STG, superior temporal gyrus; STS, superior temporal sulcus.

dipoles of spikes to be concentrated in the bilateral temporal gyrus, predominantly in the right hemisphere (-Fig. 1D). An auditory evoked field from a $1 \mathrm{kHz}$ tone burst (N100m) was absent in the right auditory cortex but remained normal in the left (- Fig. 1D and - Supplementary Figure S1 [online only]). The CSW and small spikes in the right hemisphere were suppressed by one course of IVMP and increased doses of clonazepam $(0.08 \mathrm{mg} / \mathrm{kg} / \mathrm{d})$.

The patient was taught sign language, which improved her communication skills and allowed her to learn new words and sentences. Six months after the recurrence of CSW, her verbal comprehension began to recover; she regained the ability to have simple conversations, repeat sentences, and learn new words. (At this point,14 out of 16 tasks were answered correctly in terms of accurate object names, and she also got the instructional comprehension task right at 14 out of 16 tasks). She continues to recover, and the ECD-SPECT showed almost normal perfusion pattern in both hemispheres a year after recovery (-Fig. 1C). At the 2-year follow-up from the onset of the disease, she has been able to recite simple sentences and carry-on daily conversations of preschool age, but it still takes time for her to learn complex words. The Wechsler Intelligence Scale for Children-IV (WISC-IV) test at the age of 6 was 76 for FSIQ, 76 for verbal IQ 93 for performance IQ 76 for working memory IQ, and 80 for processing speed IQ.
In addition to language functioning, psychological factors such as difficulty in concentrating, hyperactivity, and impulsivity may also have an impact on these problems. She will enroll in a school for the deaf.

\section{Discussion}

We treated a 4-year-old girl with LKS who suffered rapid language regression. Her epileptogenic zone was located in the right temporal cortex, including the primary auditory cortex. In the acute phase, the EEG showed spikes that spread synchronously in both hemispheres, suggesting that the dysfunction, including the left hemisphere, caused rapid language regression. On the other hand, after IVMP treatments, MEG and ECD-SPECT showed that the epileptogenic area was mainly in the right hemisphere, while the AEF waves from the auditory cortex remained on the left side. These findings indicated that after the first treatment, the dysfunction of the right hemisphere was strong, but the left probably retained some function.

We hypothesized that this difference in time between left and right functional recovery was related to the difference between recovery of speech and recovery of language comprehension and new word learning.

Brain dysfunction was localized by trauma and infarction studies in adults, which showed that the nondominant 
hemisphere lesion had no significant effect on language function. The question arising at this time is whether the entire function of language can remain normal in early childhood if only the right language center is impaired. Our patient showed early signs of recovery from expressive aphasia, but why did she continue to have receptive aphasia and diminished language comprehension? Unlike aphasia in adult, the right-hemisphere disorder has been reported to cause delays in language comprehension and gestures in early childhood. ${ }^{2}$ In the period of language acquisition, it requires a process of understanding what the words mean by integrating and understanding the visual, auditory, and contextual information. It is thought that the right hemisphere works predominantly with respect to its integrating role. This right-hemisphere integration processing advantage is less noticeable once word acquisition is completed to some extent, replaced by a rapid, efficient, and automatic process of mapping well-known sounds onto well-known semantic patterns. Presumably, patients with early childhood onset LKS also require this right-hemisphere function during the recovery process of lost language.

Advances in brain network research, such as diffusion tensor imaging, have led to the language acquisition theory called the dual-stream model (-Fig. 2) ${ }^{3-5}$ The first step is for the input language information to be analyzed by the bilateral dorsal STG and superior temporal sulcus for frequencytime and phonological domains.

The input language is further analyzed in two parallel routes: the dorsal and the ventral pathways. The dorsal pathway, with its strong left hemispheric dominance, centers on grammar in higher-order language functions. The ventral pathway, which has a slightly weaker left hemispheric dominance, plays a role in accessing lexical memory and processing linguistic meaning after the recognition of phonemes as language. In other words, it can be said that the input language information is initially processed in both hemispheres, and that language acquisition and generation involve collaboration between the two hemispheres, not just the dominant hemisphere.

Our patient's symptoms of auditory agnosia and language persisted even after the spike spreading to the left hemisphere had subsided, suggesting that the frequency and phonological analysis originally performed bilaterally may have been inadequate because the right hemisphere was still damaged. In fact, follow-up ECD-SPECT 1 year after relapse showed that the hyperperfusion of the right hemisphere has disappeared (-Fig. 1C), and by this time her receptive aphasia was getting much better. This course of ECD-SPECT recovery and language recovery supports our considerations. We speculate that our case, course, and neuroimaging are consistent with the dual-stream model of speech processing in which the non-dominant hemisphere also plays a major role in language comprehension, especially in early phonological processing and new word acquisition.

Conflict of Interest

None declared.

\section{References}

1 Paetau R. Magnetoencephalography in Landau-Kleffner syndrome. Epilepsia 2009;50(Suppl 7):51-54

2 Bates E. Plasticity, localization, and language development. In: Broman SH, Fletcher JM, eds. The Changing Nervous System: Neurobehavioral Consequences of Early Brain Disorders. 1999: 214-253

3 Hickok G, Poeppel D. The cortical organization of speech processing. Nat Rev Neurosci 2007;8(05):393-402

4 Metz-Lutz MN. The assessment of auditory function in CSWS: lessons from long-term outcome. Epilepsia 2009;50 (Suppl 7):73-76

5 Hoshi K, Miyazato K. Architecture of human language from the perspective of a case of childhood aphasia-Landau-Kleffner syndrome. Biolinguistics 2016;10:136-196 